\title{
Inhibitory Effect of Natural Anti-Inflammatory Compounds on Cytokines Released by Chronic Venous Disease Patient-Derived Endothelial Cells
}

\author{
Veronica Tisato, ${ }^{1}$ Giorgio Zauli, ${ }^{2}$ Erika Rimondi, ${ }^{3}$ Sergio Gianesini, ${ }^{4}$ Laura Brunelli, \\ Erica Menegatti, ${ }^{4}$ Paolo Zamboni, ${ }^{1,4}$ and Paola Secchiero ${ }^{1}$ \\ ${ }^{1}$ Department of Morphology, Surgery and Experimental Medicine and LTTA Centre, University of Ferrara, \\ Via Fossato di Mortara 66, 44100 Ferrara, Italy \\ ${ }^{2}$ Institute for Maternal and Child Health, IRCCS Burlo Garofolo, Via dell'Istria 65/01, 34137 Trieste, Italy \\ ${ }^{3}$ Department of Life Sciences, University of Trieste, Via Manzoni 16, 34137 Trieste, Italy \\ ${ }^{4}$ Vascular Disease Center, University of Ferrara, Via Aldo Moro 8, 44124 Ferrara, Italy \\ Correspondence should be addressed to Paola Secchiero; paola.secchiero@unife.it
}

Received 7 November 2013; Accepted 28 November 2013

Academic Editor: Dianne Cooper

Copyright (c) 2013 Veronica Tisato et al. This is an open access article distributed under the Creative Commons Attribution License, which permits unrestricted use, distribution, and reproduction in any medium, provided the original work is properly cited.

Large vein endothelium plays important roles in clinical diseases such as chronic venous disease (CVD) and thrombosis; thus to characterize CVD vein endothelial cells (VEC) has a strategic role in identifying specific therapeutic targets. On these bases we evaluated the effect of the natural anti-inflammatory compounds $\alpha$-Lipoic acid and Ginkgoselect phytosome on cytokines/chemokines released by CVD patient-derived VEC. For this purpose, we characterized the levels of a panel of cytokines/chemokines $(n=31)$ in CVD patients' plasma compared to healthy controls and their release by VEC purified from the same patients, in unstimulated and TNF- $\alpha$ stimulated conditions. Among the cytokines/chemokines released by VEC, which recapitulated the systemic profile (IL-8, TNF- $\alpha$, GM-CSF, INF- $\alpha 2$, G-CSF, MIP-1 $\beta$, VEGF, EGF, Eotaxin, MCP-1, CXCL10, PDGF, and RANTES), we identified those targeted by ex vivo treatment with $\alpha$-Lipoic acid and/or Ginkgoselect phytosome (GMCSF, G-CSF, CXCL10, PDGF, and RANTES). Finally, by investigating the intracellular pathways involved in promoting the VEC release of cytokines/chemokines, which are targeted by natural anti-inflammatory compounds, we documented that $\alpha$-Lipoic acid significantly counteracted TNF- $\alpha$-induced NF- $\kappa$ B and p38/MAPK activation while the effects of Ginkgo biloba appeared to be predominantly mediated by Akt. Our data provide new insights into the molecular mechanisms of CVD pathogenesis, highlighting new potential therapeutic targets.

\section{Introduction}

Although $\alpha$-Lipoic acid has been firstly explored in the light of its natural antioxidant properties and its ability to induce apoptosis in several tumour cell lines $[1,2]$, the therapeutic implications related to its clinical use have been extensively reviewed underlying overall beneficial effects on several pathological conditions such as diabetes mellitus and atherosclerosis [3, 4]. Among the putative cellular therapeutic targets of $\alpha$-Lipoic acid, also the endothelial compartment has been considered and some reports suggested a protective effect of $\alpha$-Lipoic acid on endothelial cell damage [5]. In the same fashion, Ginkgo biloba extract derivatives have been evaluated in "standard" endothelial cell models (such as human umbilical vein endothelial cells, HUVEC) as vascular protective agents to attenuate oxidative stress damage [6].

Endothelial cells derived from different anatomical districts are heterogeneous in terms of the pattern of antigens expression, secreted molecules, and immunological properties and therefore common in vitro endothelial models, such as HUVEC, cannot properly recapitulate the in vivo features of pathological endothelium. In this light, the responses of large vein endothelium are thought to play important 
roles in clinical diseases, such as chronic venous disease (CVD) and thrombosis $[7,8]$. The endothelium actively reacts in response to changes of the local environment by expressing and releasing specific cytokines, chemokines, and soluble chemical mediators that, in turn, play a role in the pathophysiology of CVD [9-12]. The therapeutic options for CVD patients range from conservative therapies, minimally invasive approaches, and surgical treatments; nevertheless CVD remains a disease with a high recurrence rate [13].

In the present study, we have addressed if the natural compounds $\alpha$-Lipoic acid and Ginkgo biloba might have a role in treating CVD through a direct effect on the pathological endothelium, by using vein endothelial cells (VEC) isolated from patients at different stages of CVD. In particular, we have evaluated the anti-inflammatory effect of the natural compounds $\alpha$-Lipoic acid and Ginkgoselect phytosome on cytokines/chemokines released by CVD patientderived VEC. For this purpose, we first characterized the systemic levels of a wide panel of cytokines/chemokines $(n=31)$ in the plasma of patients affected by CVD with respect to normal healthy controls and the release of these cytokines/chemokines by VEC purified from the same CVD patients, in unstimulated and TNF- $\alpha$-stimulated conditions. Finally, we investigated the intracellular signal transduction pathways involved in promoting the VEC release of cytokines/chemokines, which are targeted by natural antiinflammatory compounds. Overall, the data reported in the present study provide new insights into the molecular mechanisms of CVD pathogenesis, highlighting new potential therapeutic targets.

\section{Materials and Methods}

2.1. Recruitment of Patients and Samples Collection. The main demographic/clinical characteristics of CVD patients enrolled in this study are reported in (see Supplementary Table 1 in Supplementary Material available online at http://dx.doi.org/10.1155/2013/423407). Forearm blood samples of patients affected by primary CVD with superficial venous reflux (C2-4EpAsPr following the CEAP classification) were collected in the presence of sodium citrate before varicose veins surgery. Blood samples were immediately centrifuged for plasma isolation that were stored at $-80^{\circ} \mathrm{C}$ in single-use aliquots. Plasma samples collected from healthy subjects were used as controls. The surgical specimens were collected during surgery for conservative and hemodynamic treatment of venous insufficiency in ambulatory care. Highly purified VEC cultures obtained from CVD patients were isolated and characterized for morphological and phenotypic properties, as previously described [14]. The procedures followed were in accordance with the Declaration of Helsinki, approved by the institutional review board (University Hospital of Ferrara) and all participant subjects gave written informed consent.

2.2. Endothelial Cell Cultures and Treatments. Patient-derived endothelial cells were used within passages 3 to 7. Cells were grown on EGM2 medium (Lonza, Walkersville, MD) with 2\% FBS and full supplements (EGM2 Bullet kit, Lonza) in $5 \mu \mathrm{g} / \mathrm{cm}^{2}$ fibronectin precoated tissue culture plates (BD, Becton Dickinson, San Josè, CA), as described previously [15].

For endothelial cell treatments, the following reagents have been used: recombinant tumor necrosis factor (TNF)$\alpha$ (R\&D Systems, Minneapolis, MN), Ginkgoselect phytosome, and $\alpha$-Lipoic acid (kindly provided by LABOREST S.p.A., Milano, Italy) were dissolved in distilled water and in dimethylsulfoxide (DMSO, Sigma, St. Louis, MO), respectively, and at a final concentration such that the DMSO did not exceed $0.1 \%$ in the cell culture media. The optimal concentrations of TNF- $\alpha(5 \mathrm{ng} / \mathrm{mL})$, Ginkgoselect phytosome, and $\alpha$-Lipoic acid (both used at $100 \mu \mathrm{g} / \mathrm{mL}$ ) were determined in preliminary dose-response experiments. Ginkgoselect phytosome and $\alpha$-Lipoic acid were added to the cells 1 hour before treatment with TNF- $\alpha$. In selected experiments, cells were preincubated for 1 hour with pharmacologic inhibitors of ERK1/2 (PD98059; $50 \mu \mathrm{M}$; Calbiochem, La Jolla, CA), p38/MAPK (SB203580; $10 \mu \mathrm{M}$; Calbiochem), NF- $\kappa \mathrm{B}$ (Parthenolide; $10 \mu \mathrm{M}$; Enzo Life Science, Exeter, UK), and Akt (MK-2206; $5 \mu \mathrm{M}$; BioVision Incorporated, Milpitas, CA) pathways prior to the addition of TNF- $\alpha$. Cell supernatants were collected from endothelial cultures and frozen at $-80^{\circ} \mathrm{C}$ as described [16] until the cytokine analyses were performed.

\subsection{Assessment of Cell Viability and Cell Surface Inflammatory} Markers. Cell viability was monitored by light microscopic analysis of the cell monolayers after hematoxylin-eosin staining or by quantitative examination after detachment of the monolayers by means of Trypan blue dye exclusion, as described [17, 18]. The profiles of expression of the inflammatory markers ICAM-1 and VCAM-1 were analyzed by flow cytometry as previously described [19]. In brief, cells were detached with trypsin-EDTA and washed and 5 $\times 10^{5}$ cells were resuspended in $200 \mu \mathrm{L}$ of PBS containing $1 \%$ BSA (Sigma-Aldrich) and incubated for 30 minutes at $4^{\circ} \mathrm{C}$ with the following monoclonal antibodies (mAb): FITCconjugated anti-ICAM-1 (R\&D, Clone BBIG-I1) or FITCconjugated anti-VCAM-1 (R\&D, Clone BBIG-V3). Nonspecific fluorescence was assessed by incubation with isotypematched conjugated $\mathrm{mAb}[20]$.

2.4. Analysis of Cytokines and Chemokines in Plasma and Cell Culture Supernatants. Culture supernatant samples and plasma of patients were frozen and thawed only once before performing the MILLIPLEX MAP Human Cytokine/ Chemokine Panel (Merck Millipore, Billerica, MA), a beadbased multiplex immunoassay, which allows the simultaneous quantification of the following 29 human cytokines: EGF, IL-1 $\beta$, IL-1 receptor antagonist (ra), IL- $\alpha$, IL-2, IL-3, IL4, IL-5, IL-6, IL-7, IL-8, IL-10, IL-12(p40), IL-12(p70), IL13, IL-15, 1L-17A, EOTAXIN, G-CSF, GM-CSF, IFN- $\alpha 2$, IFN$\gamma$, CXCL10, MCP-1, MIP-1 $\alpha$, MIP-1 $\beta$, TNF- $\alpha$, TNF- $\beta$, and VEGF. Moreover, a custom-made MILLIPLEX MAP Human Cytokine/Chemokine Magnetic Bead Panel (Merck Millipore) was used to quantify the cytokines PDGF-AB/BB and 
TABLE 1: Circulating levels of cytokines/chemokines and growth factors in healthy controls and CVD patients.

\begin{tabular}{|c|c|c|c|}
\hline \multirow{2}{*}{ Cytokines/Chemokines } & \multicolumn{2}{|c|}{ Plasma levels $(\mathrm{pg} / \mathrm{mL})^{\mathrm{a}}$} & \multirow{2}{*}{$\begin{array}{l}\text { Limit of detection } \\
(\mathrm{pg} / \mathrm{mL})\end{array}$} \\
\hline & Controls & CVD patients & \\
\hline IL-10 & $<$ OOR & $<$ OOR & \\
\hline IL-12 (p40) & $<\mathrm{OOR}$ & $<\mathrm{OOR}$ & 1.1 \\
\hline IL-13 & $<\mathrm{OOR}$ & $<\mathrm{OOR}$ & 7.4 \\
\hline IL-15 & $<\mathrm{OOR}$ & $<\mathrm{OOR}$ & 1.3 \\
\hline IL-17A & $<\mathrm{OOR}$ & $<$ OOR & 1.2 \\
\hline IL- $1 \alpha$ & $<\mathrm{OOR}$ & $<\mathrm{OOR}$ & 0.7 \\
\hline IL- $1 \beta$ & $<\mathrm{OOR}$ & $<\mathrm{OOR}$ & 9.4 \\
\hline IL-2 & $<\mathrm{OOR}$ & $<$ OOR & 0.8 \\
\hline IL-3 & $<\mathrm{OOR}$ & $<$ OOR & 1.0 \\
\hline IL-4 & $<\mathrm{OOR}$ & $<$ OOR & 0.7 \\
\hline IL-5 & $<\mathrm{OOR}$ & $<\mathrm{OOR}$ & 4.5 \\
\hline IL-6 & $<\mathrm{OOR}$ & $<\mathrm{OOR}$ & 0.5 \\
\hline TNF- $\beta$ & $<\mathrm{OOR}$ & $<$ OOR & 0.9 \\
\hline MIP-1 $\alpha$ & $0(0.87 \pm 2)$ & $13(13.7 \pm 14.5)^{* *}$ & 1.5 \\
\hline IL-8 & $2.2(2.8 \pm 1.8)$ & $14.4(25.8 \pm 35.5)^{*}$ & 2.9 \\
\hline IL-7 & $2.7(2.9 \pm 1.3)$ & $3.7(3.5 \pm 1.3)$ & 0.4 \\
\hline IFN- $\gamma$ & $3.18(3.7 \pm 1.9)$ & $2.5(4.4 \pm 3.8)$ & 1.4 \\
\hline IL-12 (p70) & $3.2(3.8 \pm 2)$ & $3.5(3.7 \pm 1.3)$ & 0.8 \\
\hline TNF- $\alpha$ & $3.3(3.8 \pm 1.6)$ & $6.9(6.8 \pm 3.3)^{* *}$ & 0.6 \\
\hline GM-CSF & $4.6(4.9 \pm 1.9)$ & $5.9(6.7 \pm 3)^{*}$ & 0.7 \\
\hline IFN- $\alpha 2$ & $9.5(12 \pm 8.3)$ & $15(17.7 \pm 7.3)^{*}$ & 7.5 \\
\hline G-CSF & $16.9(17.9 \pm 6.8)$ & $29.2(28.1 \pm 18.1)^{\S}$ & 2.9 \\
\hline IL-1RA & $18.3(20.3 \pm 10)$ & $16.7(20.2 \pm 11.5)$ & 1.8 \\
\hline MIP-1 $\beta$ & $19.6(20.2 \pm 6.8)$ & $50(54.5 \pm 35.7)^{* *}$ & 8.3 \\
\hline VEGF & $29.2(34.8 \pm 17)$ & $64.6(63.4 \pm 33.5)^{* *}$ & 3.0 \\
\hline EGF & $36.6(40 \pm 23.3)$ & $95.4(155.5 \pm 152.5)^{*}$ & 26.3 \\
\hline Eotaxin & $48.6(54.9 \pm 26)$ & $251(260 \pm 211.7)^{* *}$ & 2.8 \\
\hline MCP-1 & $161(162.5 \pm 56)$ & $224(276.2 \pm 146.5)^{*}$ & 4.0 \\
\hline CXCL10 & $195(203.5 \pm 67.8)$ & $244(312.5 \pm 178.9)^{\S}$ & 1.9 \\
\hline PDGF & $2,770(5,476 \pm 5,027)$ & $9,369(17,352 \pm 20,163)^{*}$ & 8.6 \\
\hline RANTES & $10,414(16,126 \pm 15,567)$ & $45,399(58,283 \pm 59,353)^{*}$ & 2.2 \\
\hline
\end{tabular}

${ }^{\mathrm{a}}$ Values are expressed as median (mean $\pm \mathrm{SD}$ ); $<\mathrm{OOR}$ : out (below) of detection range. ${ }^{* *} P<0.01 ;{ }^{*} P<0.05 ;{ }^{\S} P<0.06$.

RANTES. Culture supernatant samples were processed following the manufacturer's recommended protocols and read on a MAGPIX instrument equipped with the MILLIPLEXAnalyst Software using a five-parameter nonlinear regression formula to compute sample concentrations from the standard curves.

2.5. Phosphoproteins Profile and Western Blot in Endothelial Cells. The MILLIPLEX MAP Human Multi-Pathway 9plex Magnetic Bead Signalling kit phosphoprotein was used to detect changes in phosphorylated ERK/MAP kinase 1/2 (Thr185/Tyr187), Akt (Ser473), STAT3 (Ser727), JNK (Thr183/Tyr185), p70 S6 kinase (Thr412), NF- $\kappa$ B (Ser536), STAT5A/B (Tyr694/699), CREB (Ser133), and p38 (Thr180/Tyr182) in endothelial cell lysates using the Luminex system, according to the manufacturer's instructions. Briefly, endothelial cells were plated in $100 \mathrm{~mm}$ dishes, grown at subconfluence, and subjected to partial FCS reduction (to $0.5 \%$ ) for 18 hours before the addition of treatments. Cells were harvested in MILLIPLEX MAP lysis buffer in the presence of Protease Inhibitor Cocktail Set III (Calbiochem). Each lysate (20 $\mu \mathrm{g}$ total protein) was diluted in MILLIPLEX MAP Assay Buffer 2, incubated at $4^{\circ} \mathrm{C}$ overnight, and analyzed according to the manufacturer's instructions. The Median Fluorescence Intensity (MFI) was measured with the Luminex System.

To validate the MILLIPLEX MAP human multipathway data, we have performed Western blot analyses as previously described $[21,22]$. Briefly, cells were harvested in lysis buffer containing 1\% Triton X-100, $150 \mathrm{mM} \mathrm{NaCl}, 50 \mathrm{mM}$ Tris- $\mathrm{HCl}$ pH 6.8, and protease inhibitors (protease inhibitor cocktail; 
TABLE 2: Baseline release of cytokines/chemokines and growth factors by pathological VEC.

\begin{tabular}{lcc}
\hline $\begin{array}{l}\text { Cytokines/ } \\
\text { Chemokines }\end{array}$ & $\begin{array}{c}\text { Release levels } \\
(\mathrm{pg} / \mathrm{mL} / 24 \text { hours })^{\mathrm{a}}\end{array}$ & $\begin{array}{c}\text { Limit of detection } \\
(\mathrm{pg} / \mathrm{mL})\end{array}$ \\
\hline MIP-1 $\alpha$ & $<$ OOR & 2.9 \\
IL-8 & $2,616(3,111.8 \pm 2,532.4)$ & 0.4 \\
IL-7 & $11.6(16.7 \pm 10.1)$ & 1.4 \\
IFN- $\gamma$ & $2.1(2.3 \pm 0.9)$ & 0.8 \\
IL-12 $(\mathrm{p} 70)$ & $2.8(2.8 \pm 0.4)$ & 0.6 \\
TNF- $\alpha$ & $1.8(1.6 \pm 0.3)$ & 0.7 \\
GM-CSF & $8.2(8.6 \pm 5.5)$ & 7.5 \\
IFN- $\alpha 2$ & $9.1(9.3 \pm 3.7)$ & 2.9 \\
G-CSF & $49(65.9 \pm 37)$ & 1.8 \\
IL-1RA & $10.4(11.4 \pm 4.2)$ & 8.3 \\
MIP-1 $\beta$ & $4(4.5 \pm 2.1)$ & 3.0 \\
VEGF & ND & 26.3 \\
EGF & ND & 2.8 \\
Eotaxin & $10.2(15.1 \pm 8.5)$ & 4.0 \\
MCP-1 & $8,663(8,630.1 \pm 1,450)$ & 1.9 \\
CXCL10 & $20.5(41.6 \pm 55)$ & 8.6 \\
PDGF & $1,399(1,494.6 \pm 468.9)$ & 2.2 \\
RANTES & $31(33.3 \pm 14.9)$ & 1.2 \\
\hline
\end{tabular}

${ }^{a}$ Values are expressed as median (mean $\pm \mathrm{SD}$ ); <OOR: out (below) of detection range; ND: not determinable because component of the endothelial medium.

Roche Diagnostics). Protein determination was performed by Bradford assay (Bio-Rad, Richmond, CA). Equal amounts of protein $(50 \mu \mathrm{g})$ for each sample were migrated in acrylamide gels, blotted onto nitrocellulose filters, and probed with the following antibodies for the phosphorylated forms of ERK1/2, p38/MAPK, and IkB and for the respective total protein kinase content for verifying loading evenness: anti-phosphop44/42 MAPK (Erk1/2) and anti-p44/42 MAPK (Erk1/2) (both from Cell Signalling Technology, Danvers, MA); antiphospho-p38/MAPKinase and anti-p38/MAPKinase (both from Cell Signaling Technology, Beverly, MA); anti-phosphoAkt and anti-Akt (both from Merck Millipore); anti-IkB (from Santa Cruz Biotechnology, Santa Cruz, CA) and antitubulin (Sigma-Aldrich). After incubation with peroxidaseconjugated anti-mouse and anti-rabbit IgG, specific reactions were revealed with the ECL Lightning detection kit (Perkin Elmer, Boston, MA). Densitometry values were estimated by the ImageQuant TL software (GE Healthcare Bio-Sciences $A B$, Uppsala, Sweden). Multiple film exposures were used to verify the linearity of the samples analyzed and avoid saturation of the film.

2.6. Statistical Analysis. Descriptive statistics were calculated. For each set of experiments, values were reported as means \pm standard deviation (SD). The results were evaluated by using Student's $t$-test and the Mann-Whitney rank-sum test, when appropriate. All statistical analyses were performed with SPSS
Statistic 20 software (SPSS Inc., Chicago, IL). $P$ values were considered significant when $<0.05$.

\section{Results}

3.1. Characterization of the Plasmatic Levels of Cytokines and Chemokines in CVD Patients and Potential Contribution of Venous Endothelium. In the first set of experiments, we sought to investigate the pattern of circulating cytokines/chemokines in CVD patients. For this purpose, plasma samples of CVD patients were collected before surgery for conservative and hemodynamic treatment of venous insufficiency in ambulatory care and analyzed for a panel of 31 cytokines/chemokines involved in the inflammatory and/or thrombosis processes. Overall, CVD patients (mean age of $52.3 \pm 11.5$ ) were characterized by a mean duration of the disease of $19.3 \pm 11.6$ years; besides $73 \%$ of them showed a family history of CVD with a variable incidence of other relevant comorbidities such as diabetes mellitus, hypertension, hypercholesterolemia, and cardiac disease (Supplementary Table 1). Among the 31 cytokines/chemokines analyzed by multiplex assay, 18 were detectable in plasma samples of both CVD patients and healthy control individuals: MIP- $1 \alpha$, IL-8, IL-7, IFN- $\gamma$, IL12 (p70), TNF- $\alpha$, GM-CSF, IFN- $\alpha 2$, G-CSF, IL-1RA, MIP- $\beta$, VEGF, EGF, Eotaxin, MCP-1, CXCL10, PDGF, and RANTES (Table 1). Of interest, within the panel of cytokines and chemokines detectable at the plasma level, 12 (MIP- $1 \alpha$, IL8 , TNF- $\alpha$, GM-CSF, IFN- $\alpha 2$, MIP- $1 \beta$, VEGF, EGF, Eotaxin, MCP-1, PDGF, and RANTES) were significantly $(P<0.05)$ increased in CVD patients compared to healthy controls while 2 additional cytokines (G-CSF and CXCL10) showed levels close to significance (Table 1). These data suggested the potential role of these cytokines/chemokines in the pathogenesis and/or progression of the disease.

To evaluate the role of pathological endothelium in the establishment of the increased levels of circulating cytokines/chemokines characterizing CVD, we have isolated pathological VEC from surgical specimens obtained from the same CVD patients analysed for the circulating cytokines/chemokines (Supplementary Table 1 and Table 1). VEC cultures were characterized and defined as $\mathrm{CD}^{+} 1^{+} / \mathrm{CD} 105^{+} / \mathrm{CD} 146^{+} / \mathrm{CD} 144^{+} / \mathrm{CD} 45^{-} / \mathrm{CD} 14^{-}$cells, as previously described [9], and were assessed for the baseline release of the same panel of 31 cytokines/chemokines evaluated in plasma samples of CVD patients. In this respect, it should be noticed that since VEGF and EGF are essential components of the VEC culture medium, it was not possible to distinguish exogenously added VEGF and EGF from endogenously produced cytokines. Of interest, among the cytokines/chemokines detectable at the plasma of CVD patients (Table 1), only MIP- $1 \alpha$ was undetectable in VEC culture supernatants (Table 2), while IL-8, IL-7, IFN- $\gamma$, IL$12(\mathrm{p} 70)$, TNF- $\alpha$, GM-CSF, IFN- $\alpha 2$, G-CSF, IL-1RA, MIP$1 \beta$, Eotaxin, MCP-1, CXCL10, PDGF, and RANTES were released at different levels (Table 2). This group of data suggests that VEC represent a major cell type contributing to 


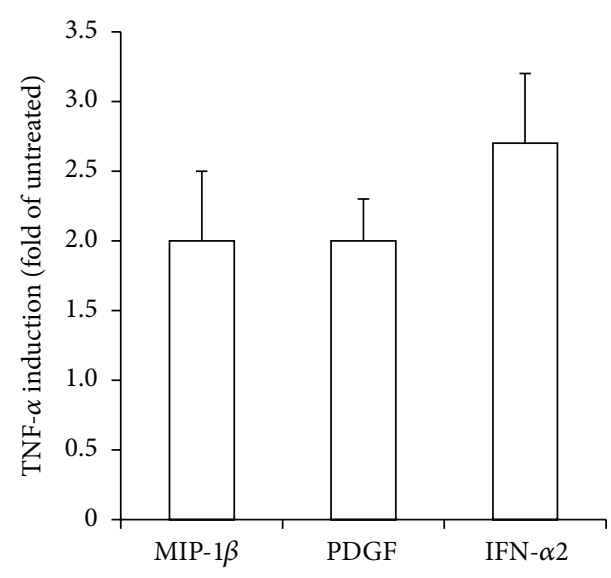

(a)

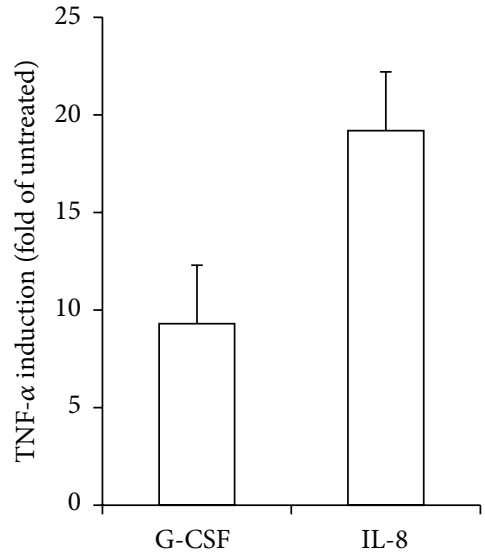

(b)

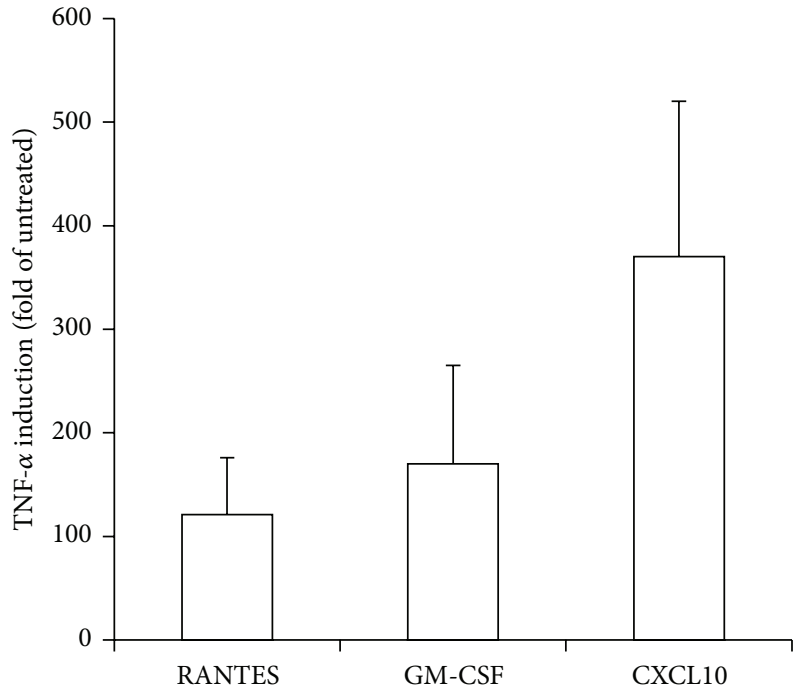

(c)

FIGURE 1: Cytokines/chemokines in vitro release by pathological VEC in response to TNF- $\alpha$. Endothelial cells were exposed for 24 hours to TNF- $\alpha(5 \mathrm{ng} / \mathrm{mL})$ before analysis of cytokines/chemokines release on culture supernatants. Results are expressed as fold of induction with respect to untreated cultures (set to 1 ). Only cytokines/chemokines showing fold of induction $\geq 2$ have been reported. Data are means \pm SD of results obtained in VEC from all CVD patients.

the production of plasmatic cytokines/chemokines in CVD patients.

In light of the fact that pathological VEC are exposed in vivo to a proinflammatory milieu $[7-11,14]$ and that we found a significant $(P<0.01)$ elevation of the plasmatic levels of TNF- $\alpha$ in CVD patients, in further experiments VEC cultures were exposed in vitro to recombinant TNF$\alpha$ in order to mimic the in vivo inflammatory microenvironment. Only those cytokines/chemokines showing a mean increase $\geq 2$-fold were considered (Figure 1). These eight cytokines/chemokines were grouped on the basis of the fold increase: MIP-1 $\beta$, PDGF, and IFN- $\alpha 2$ (between 2 and 3 mean fold increase), G-CSF and IL-8 (between 10 and 20 mean fold increase), and GM-CSF, CXCL10, and RANTES (between 100 and 300 mean fold increase) (Figure 1).
3.2. The Release of Specific Cytokines/Chemokines by Pathological VEC Is Differentially Inhibited by $\alpha$-Lipoic Acid and Ginkgoselect Phytosome. In the next set of experiments, we have investigated whether the anti-inflammatory natural compounds $\alpha$-Lipoic acid [23-28] and Ginkgo biloba derivatives $[29,30]$ affected the release of cytokines/chemokines by pathological VEC. Indeed, although it has already been reported that both compounds, and in particular $\alpha$-Lipoic acid, exhibit anti-inflammatory activities both in vitro and in vivo in the context of endothelial biology of different pathologies [23-27], it remains to be established whether these compounds are active also on pathological VEC isolated from CVD patients. Since both compounds have been shown to potentially affect the survival of endothelial cells [31-35], we started by analysing the morphology and viability of VEC 


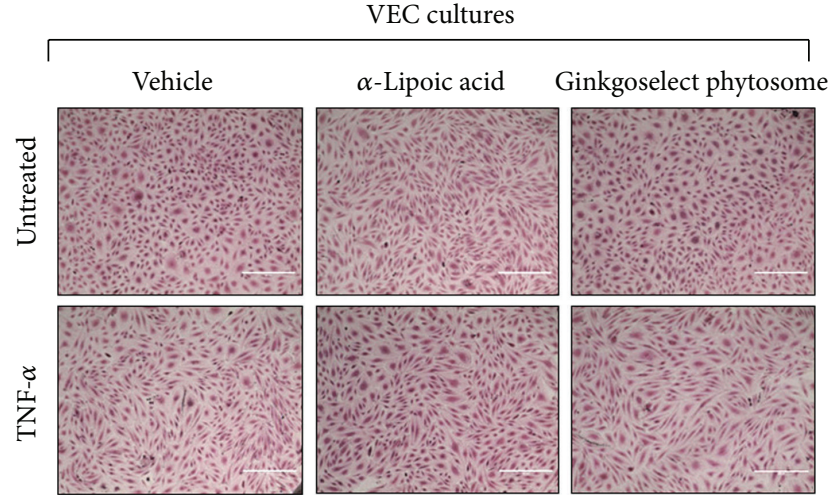

(a)

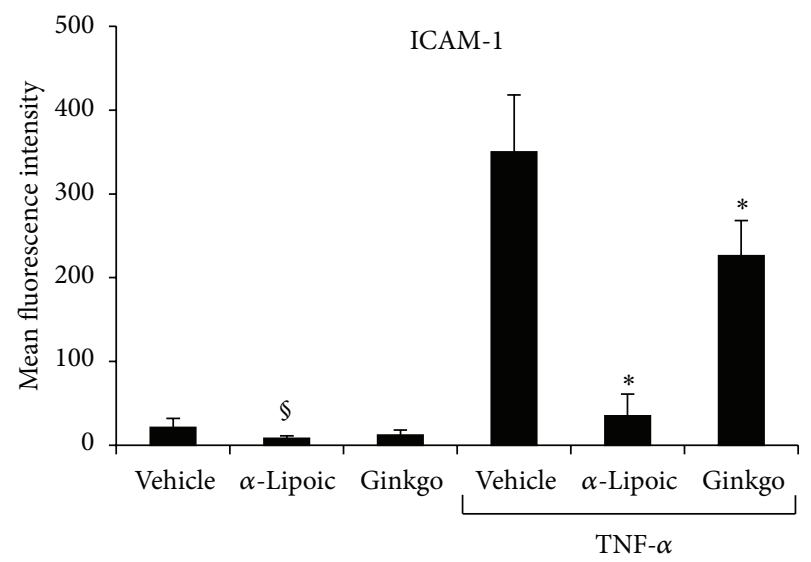

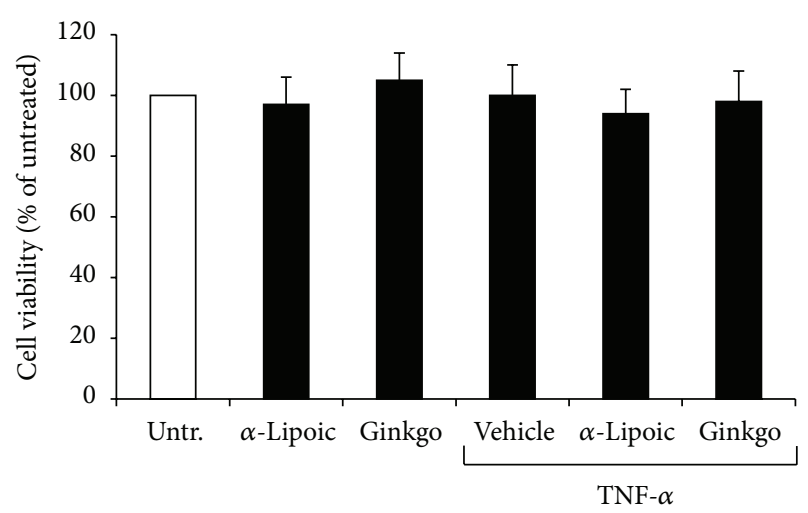

(b)

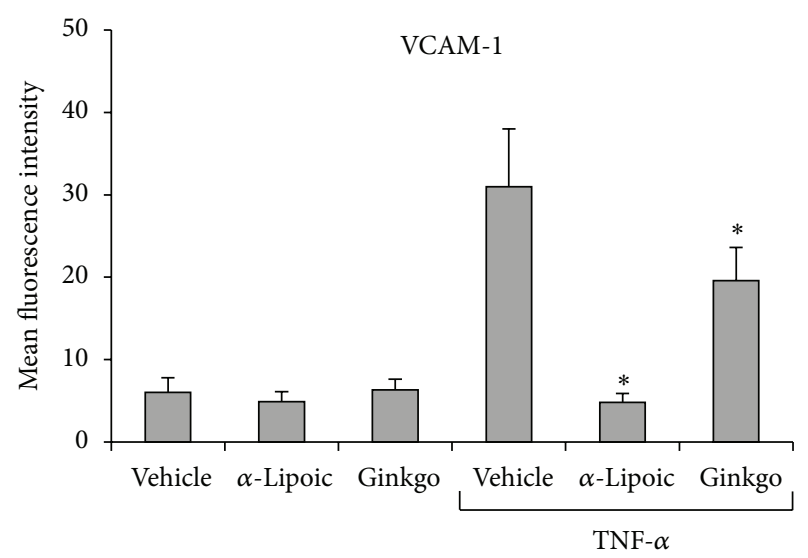

(c)

FIGURE 2: Lack of cell toxicity and anti-inflammatory properties of $\alpha$-Lipoic acid and Ginkgoselect phytosome in CVD-VEC model. Endothelial cells were exposed to TNF- $\alpha(5 \mathrm{ng} / \mathrm{mL})$, Ginkgoselect phytosome, and $\alpha$-Lipoic acid (both at $100 \mu \mathrm{g} / \mathrm{mL}) \mathrm{used}$ either alone or in combination ( 1 hour of pretreatment with Ginkgoselect phytosome and $\alpha$-Lipoic acid before addition of TNF- $\alpha$ ) and analyzed after 24 hours of treatments. In (a), representative fields of cultures treated as indicated were observed by light microscopy after haematoxylin and eosin staining. In (b), cell viability is shown as percentage of untreated cultures (Untr.) set to $100 \%$. Data are reported as means \pm SD of three independent experiments. In (c), data are expressed as mean fluorescence intensity (MFI) after subtraction of background fluorescence from vehicle-treated cells. Results are reported as means \pm SD of four independent experiments. ${ }^{*} P<0.05$ compared to vehicle and TNF- $\alpha$ treated VEC; ${ }^{\$} P<0.05$ compared to vehicle treated VEC.

exposed to different concentrations (up to $250 \mu \mathrm{g} / \mathrm{mL}$ for each compound) of $\alpha$-Lipoic acid and Ginkgoselect phytosome for 24 hours. As shown in Figure 2(a), morphological analysis did not reveal toxic effects at the concentration of $100 \mu \mathrm{g} / \mathrm{mL}$, when compounds were used either alone or together with recombinant TNF- $\alpha$. The lack of toxicity indicated by morphological evidences was confirmed by quantitative cell viability analysis (Figure 2(b)). In addition, preexposure for 1 hour to both $\alpha$-Lipoic acid and Ginkgoselect phytosome (both used at $100 \mu \mathrm{g} / \mathrm{mL}$ ), before addition of recombinant TNF- $\alpha$ for 24 hours, significantly $(P<0.05)$ inhibited the TNF- $\alpha$ mediated induction of the proinflammatory markers VCAM-1 and ICAM-1 (Figure 2(c)). Therefore, concentration of $100 \mu \mathrm{g} / \mathrm{mL}$ for both compounds was selected for further experiments. Indeed, having confirmed that both $\alpha$-Lipoic acid and Ginkgoselect phytosome exerted anti-inflammatory activities also in pathological VEC without inducing a specific toxicity (Figure 2), we analyzed the effect of both compounds on the specific release of cytokines/chemokines by pathological VEC. As shown in Figure 3(a), in vitro treatment with $\alpha$-Lipoic acid alone was able to significantly decrease the baseline levels of PDGF, RANTES, and CXCL10, while Ginkgoselect phytosome alone only inhibited basal PDGF. In addition, pretreatment (for 1 hour) with both $\alpha$ Lipoic acid and Ginkgoselect phytosome before addition of TNF- $\alpha$ significantly downregulated the release of TNF- $\alpha$ induced PDGF, RANTES, and CXCL10 (Figure 3(b)), while $\alpha$-Lipoic acid, but not Ginkgoselect phytosome, significantly inhibited the TNF- $\alpha$-induced release of G-CSF and GMCSF (Figure 3(b)). Thus, $\alpha$-Lipoic acid exhibited a broader spectrum of inhibitory activity as compared to Ginkgoselect phytosome. On the other hand, the release of the other cytokines/chemokines upregulated by TNF- $\alpha$ (IFN- $\alpha 2$, MIP$1 \beta$, and IL-8) was unaffected by either $\alpha$-Lipoic acid or Ginkgoselect phytosome (data not shown).

3.3. Different Intracellular Signaling Pathways Mediate the Cytokine/Chemokine Release by Pathological VEC. In the last 

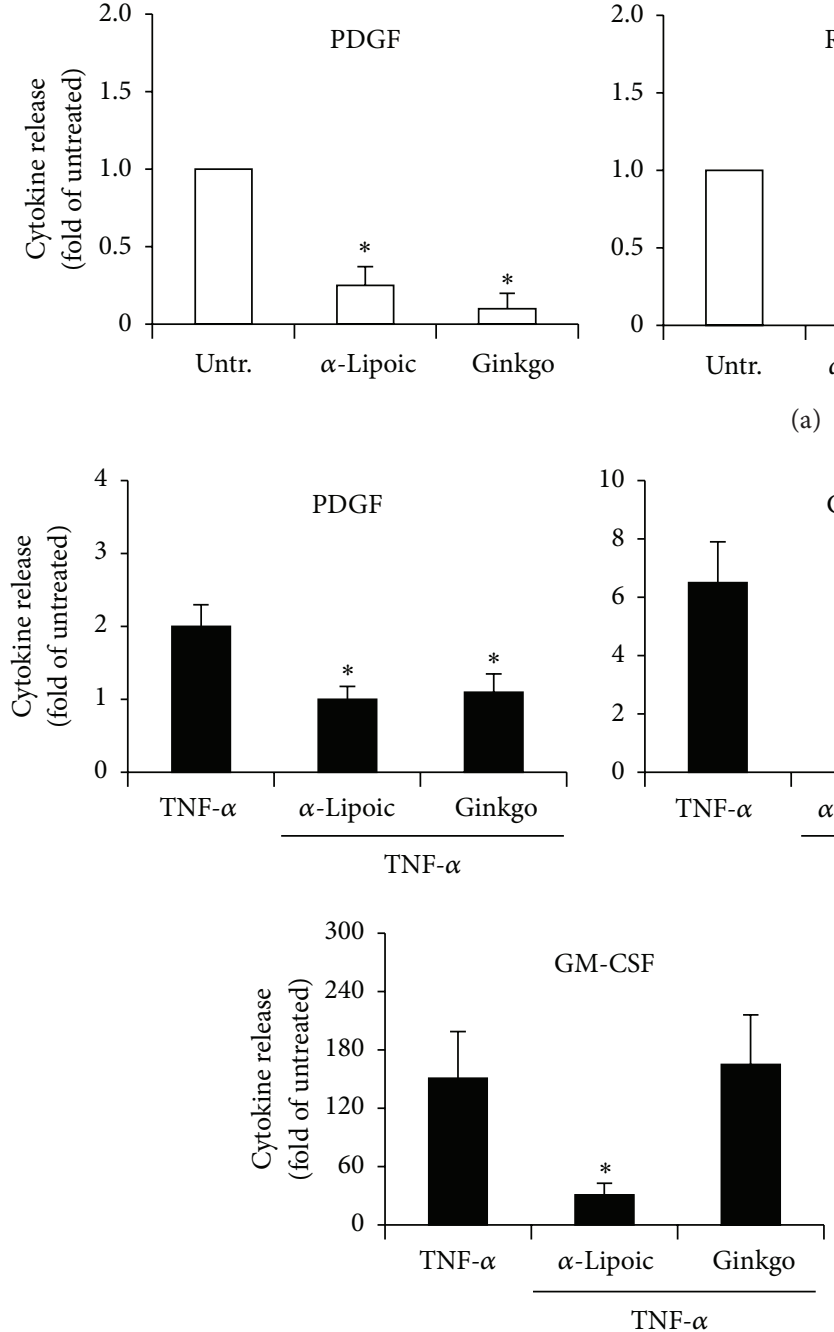

RANTES

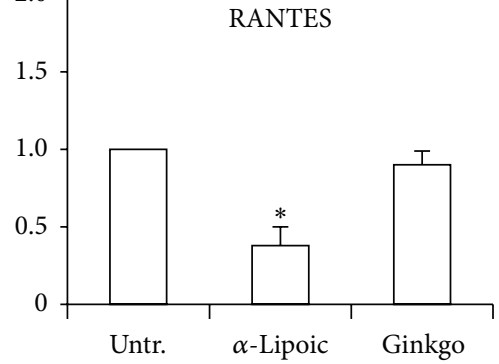

(a)

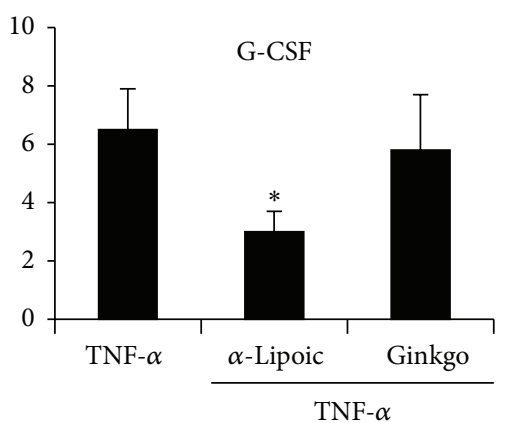

TNF- $\alpha$

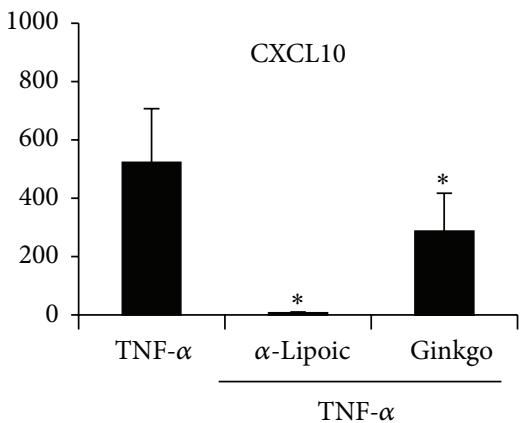

TNF- $\alpha$

(b)
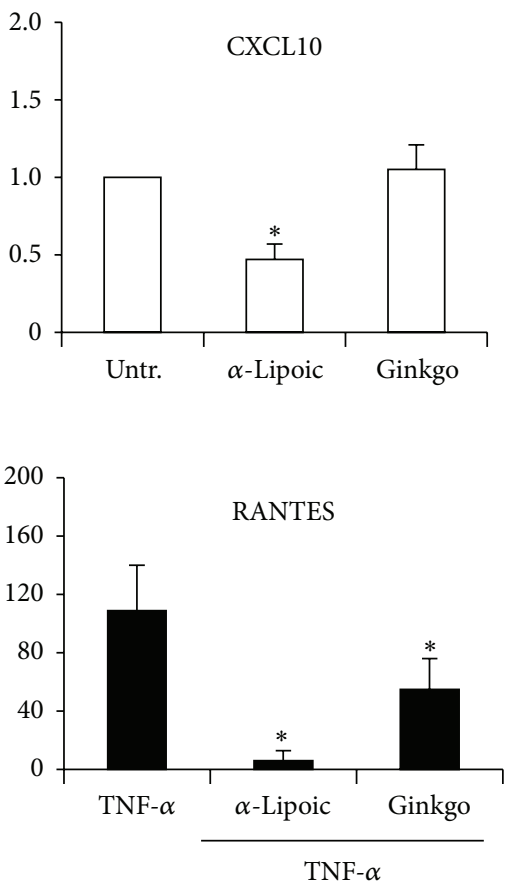

FIGURE 3: Inhibitory effects on cytokines/chemokines release of $\alpha$-Lipoic acid and Ginkgoselect phytosome in pathological VEC cultures. Cytokines/chemokines levels were measured in culture supernatants of endothelial cells treated for 24 hours with $\alpha$-Lipoic acid or Ginkgoselect phytosome used alone (a) or in combination with TNF- $\alpha$ ( 1 hour of pretreatment with $\alpha$-Lipoic acid or Ginkgoselect phytosome before addition of TNF- $\alpha$ ) (b). Results are expressed as fold of induction with respect to the untreated cultures (set to 1). Data are expressed as means \pm SD. ${ }^{*} P<0.05$ compared to untreated (a) or TNF- $\alpha$ treated cultures (b).

group of experiments, we have investigated the intracellular signalling pathways mediating the release of the various cytokines and chemokines in the culture supernatants of pathological VEC and targeted by $\alpha$-Lipoic acid and/or Ginkgoselect phytosome. In line with previous reports showing activation of various intracellular pathways by TNF- $\alpha$ treatment [36], we found that exposure of VEC cultures to recombinant TNF- $\alpha$ resulted in a time-dependent activation/phosphorylation of several signalling pathways such as JNK, p38/MAPK, ERK1/2, NF- $\kappa$ B and Akt, STAT3, and CREB (Figure 4(a)). Of note, $\alpha$-Lipoic acid significantly inhibited both NF- $\kappa \mathrm{B}$ and $\mathrm{p} 38 / \mathrm{MAPK}$ pathways at two different time points (Figure 4(b)). On the other hand, Ginkgoselect phytosome significantly inhibited p38/MAPK and Akt pathways at two different time points, while it was less efficient than $\alpha$-Lipoic acid in inhibiting NF- $\kappa \mathrm{B}$.
Neither of the two compounds was effective in modulating the remaining pathways analysed and shown in Figure 4(a). Based on the data illustrated above, the evidence that $\alpha$ Lipoic acid was more powerful than Ginkgoselect phytosome in inhibiting the release of cytokines and chemokines, either spontaneously or in response to TNF- $\alpha$, suggested that NF$\kappa \mathrm{B}$ pathway plays a primary role in promoting the TNF$\alpha$ mediated induction of cytokines and chemokines. To confirm this issue, in the last group of experiments, cells were preincubated with pharmacologic inhibitors specific for NF$\kappa \mathrm{B}, \mathrm{p} 38 / \mathrm{MAPK}, \mathrm{Akt}$, and ERK1/2 pathways 1 hour before TNF- $\alpha$ addition. As shown in Figure 5, the release of PDGF, G-CSF, RANTES, GM-CSF, and CXCL10 was significantly reduced by Parthenolide, an inhibitor of NF- $\kappa \mathrm{B}$ pathway, which showed an overlapping pattern of inhibition with $\alpha$ Lipoic acid (Figure 3), thus confirming the prominent role 

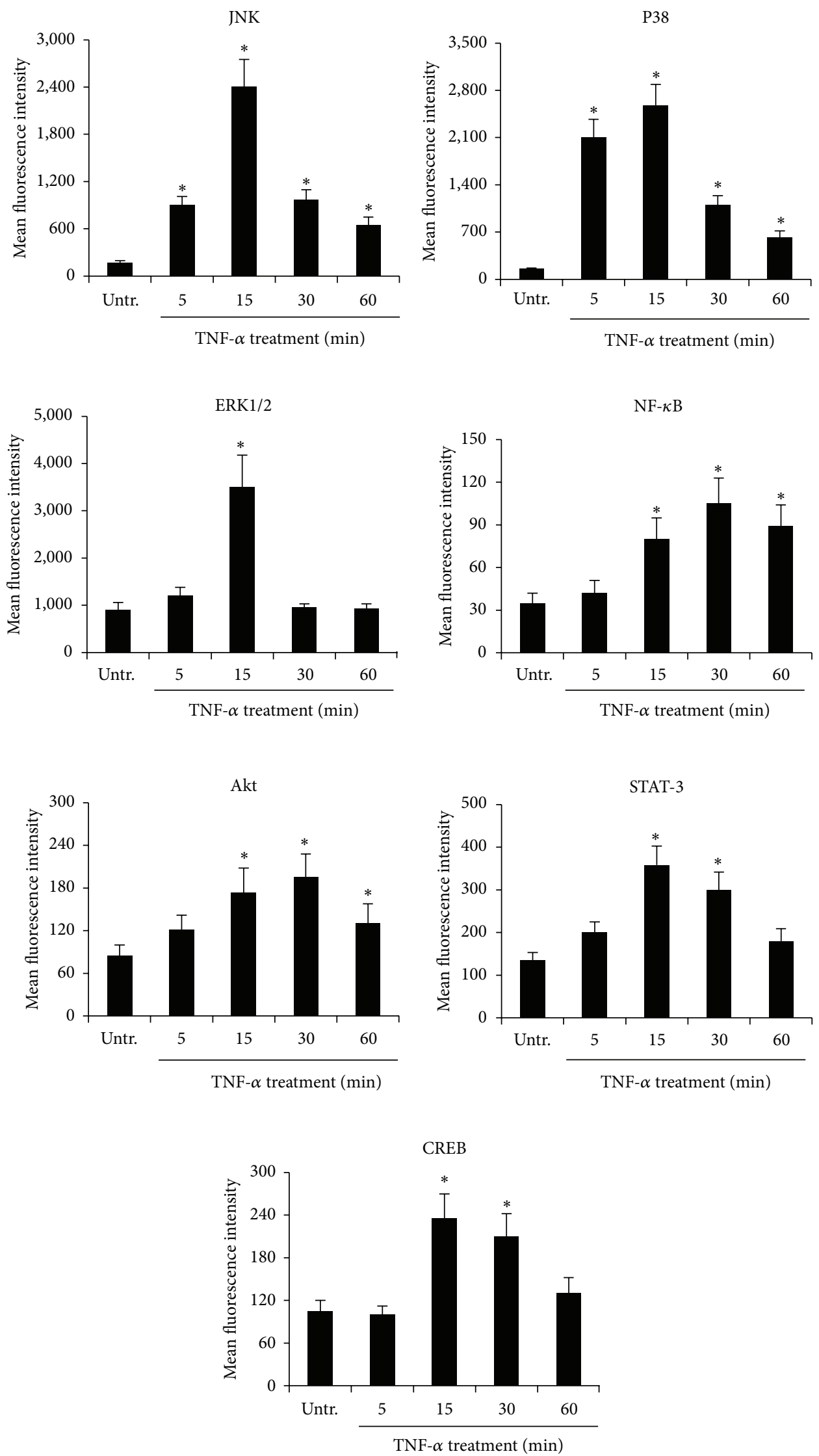

(a)

Figure 4: Continued. 

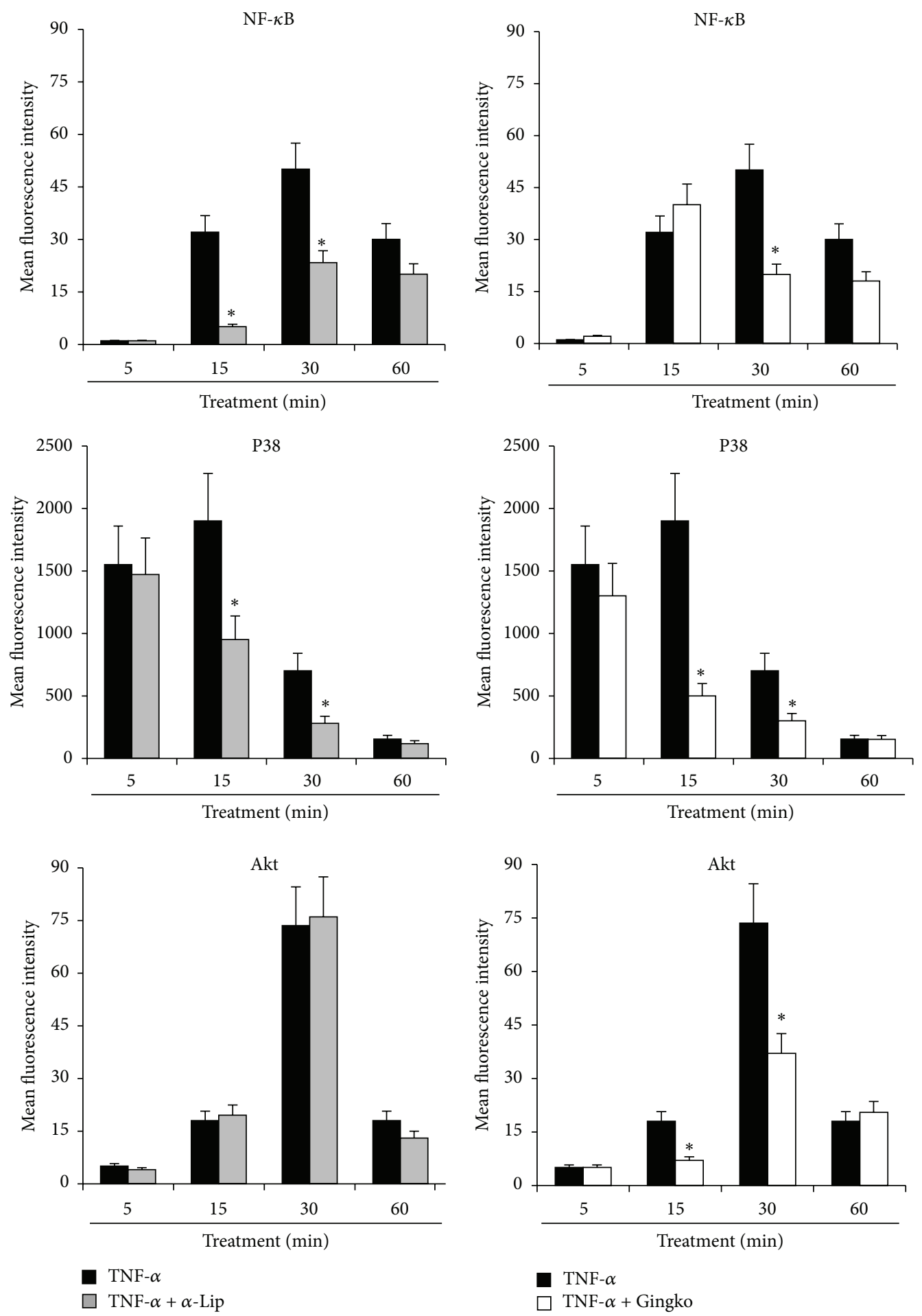

(b)

FIGURE 4: Multiplex analysis of molecular pathways activated in CVD-VEC in response to TNF- $\alpha$ stimulation: modulatory effect of $\alpha$-Lipoic acid Ginkgoselect phytosome. Endothelial cells were left untreated (Untr.) or treated with TNF- $\alpha$ ( $5 \mathrm{ng} / \mathrm{mL})$ alone (a) or in combination with $\alpha$-Lipoic acid and Ginkgoselect phytosome (preincubation for 1 hour with $\alpha$-Lipoic acid and Ginkgoselect phytosome both at $100 \mu \mathrm{g} / \mathrm{mL}$ before addition of TNF- $\alpha$; (b)) before analysis of phosphoprotein levels performed by Luminex system and expressed as mean fluorescent intensity (MFI). In (a), endothelial cells were treated with TNF- $\alpha$ for the indicated time points and cell lysates were assayed for phosphoproteins activation. ${ }^{*} P<0.05$ compared to untreated cells. In (b), VEC cultures were pretreated for 1 hour with $\alpha$-Lipoic acid and Ginkgoselect phytosome prior to stimulation with TNF- $\alpha$ for the indicated time points. Cell lysates were assayed for phosphoproteins induction and results expressed as means $\pm \mathrm{SD}$ of samples assayed in duplicate in four independent experiments. ${ }^{*} P<0.05$ compared to cells treated with TNF- $\alpha$ alone. 

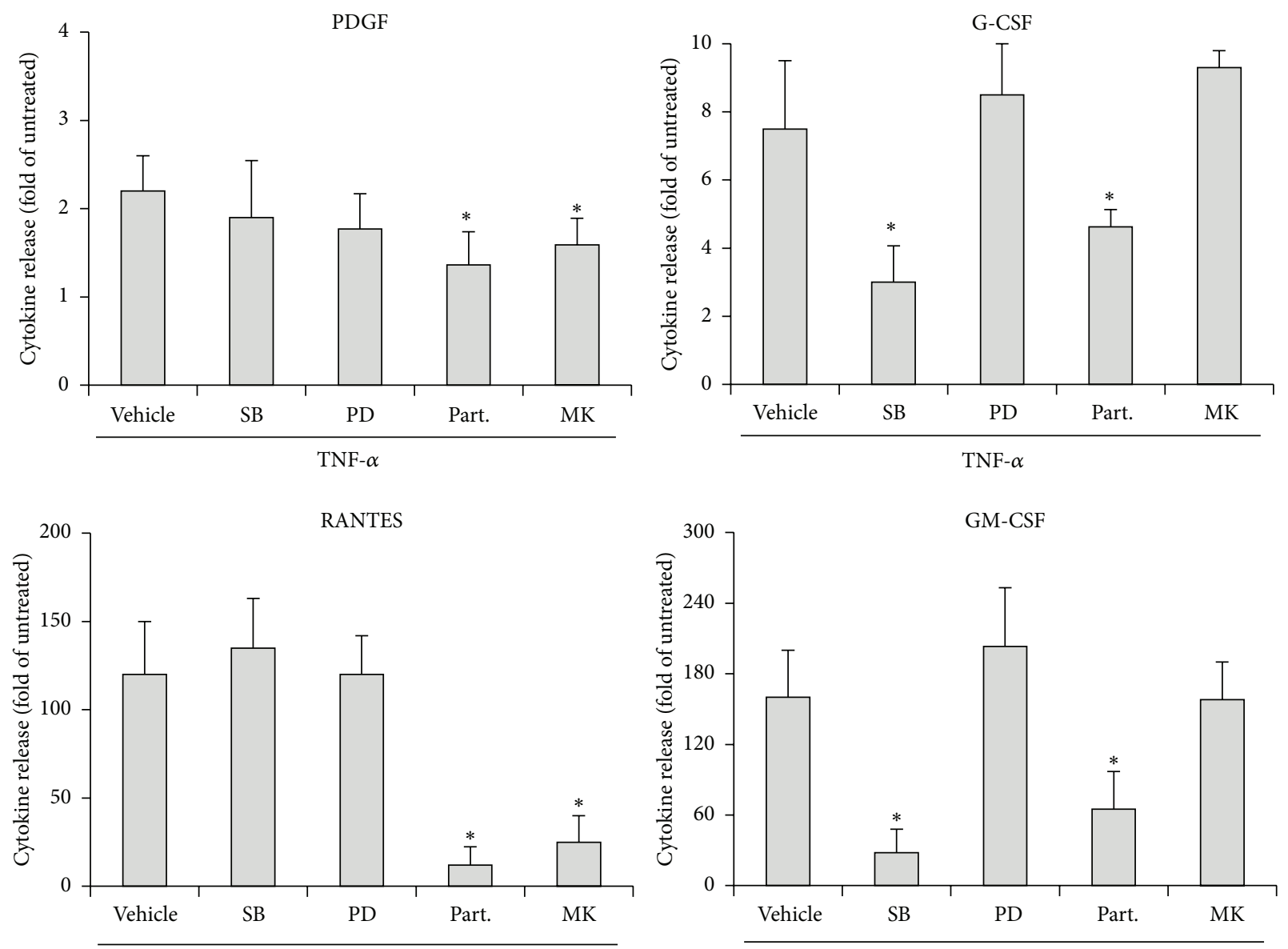

TNF- $\alpha$

TNF- $\alpha$

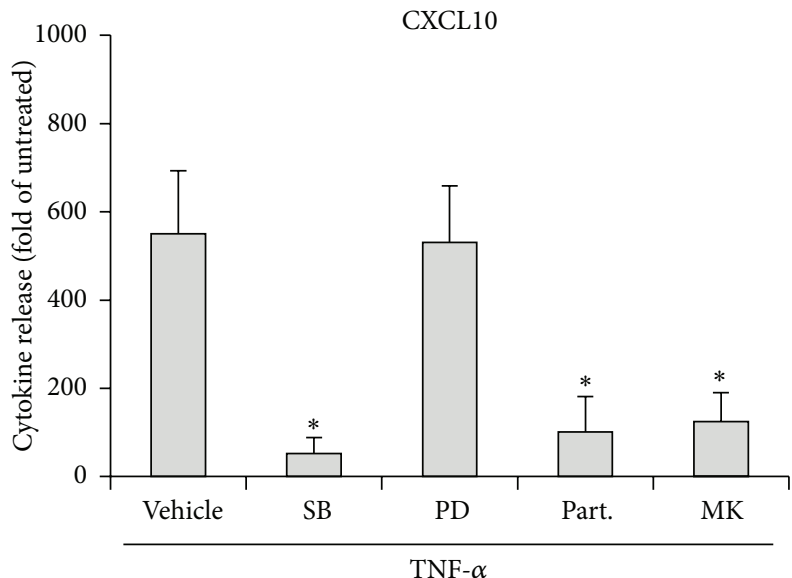

FIGURE 5: Differential effects of pharmacological inhibitors on cytokines/chemokines release in pathological VEC in response to TNF- $\alpha$. VEC were pretreated for 1 hour with SB203580 $(10 \mu \mathrm{M})$, PD98059 $(50 \mu \mathrm{M})$, Parthenolide $(10 \mu \mathrm{M})$, SP600125 $(5 \mu \mathrm{M})$, and MK-2206 (5 $\mu \mathrm{M})$ before addition of TNF- $\alpha(5 \mathrm{ng} / \mathrm{mL})$. After 24 hours, cell culture supernatants were analyzed for cytokines/chemokines release and normalized for cell number. Results are expressed as fold of induction with respect to untreated cultures (set to 1). Data are means \pm SD of results obtained from four different experiments. ${ }^{*} P<0.05$ compared to TNF- $\alpha$ /vehicle treated cells.

of NF- $\kappa \mathrm{B}$ in mediating the TNF- $\alpha$-induced release of several cytokines/chemokines as well the role of NF- $\kappa \mathrm{B}$ as the major intracellular pathway targeted by $\alpha$-Lipoic acid. Interestingly, the selective inhibitor of the p38/MAPK pathway SB203580 also significantly inhibited the release of G-CSF, GM-CSF, and CXCL10, suggesting that this pathway might also contribute to the release of cytokines/chemokines by VEC. Finally, the inhibitor of the Akt pathway induced a selective inhibition of PDGF, RANTES, and CXCL10 (Figure 5), showing a clear-cut overlapping with the inhibitory activity of 
Ginkgoselect phytosome (Figure 3). Consistently, Akt pathway represented a major target of Ginkgoselect phytosome (Figure 4(b)). The specificity of the different inhibitors of the pathways activated by TNF- $\alpha$ has been validated by Western blot, (see Supplementary Figure 1 in Supplementary Material available online).

\section{Discussion}

By analysing a large panel of cytokines/chemokines $(n=$ 31), we demonstrated for the first time that patients affected by CVD are characterized by increased plasmatic levels of MIP- $1 \alpha$, IL- 8 , TNF- $\alpha$, GM-CSF, IFN- $\alpha 2$, G-CSF, MIP$1 \beta$, VEGF, EGF, Eotaxin, MCP-1, CXCL10, PDGF, and RANTES with respect to healthy control subjects. Since the inflammatory properties of vein endothelium in CVD are poorly understood, an important point of our study was that all these cytokines, with the exception of MIP$1 \alpha$, were spontaneously released in vitro by VEC purified from the same CVD patients analyzed for the plasmatic levels of cytokines/chemokines. An additional interesting finding was that the majority of the cytokines/chemokines found elevated in plasma of CVD patients and released spontaneously in the culture supernatants by VEC obtained from CVD patients (with the exception of VEGF and EGF that were not valuable since they were contained in the endothelial culture medium) were significantly induced by recombinant TNF- $\alpha$, a major proinflammatory cytokine. Taken together, the in vivo and in vitro data suggested that a specific pattern of cytokines/chemokines (MIP-1 $\beta$, PDGF, IFN- $\alpha 2$, G-CSF, IL-8, RANTES, GM-CSF, and CXCL10) (i) is produced by pathological VEC obtained from CVD patients, (ii) is significantly increased in response to TNF- $\alpha$, and (iii) is elevated in the plasma of CVD patients. These findings suggest that pathological VEC significantly contribute to the elevation of the majority of plasmatic cytokines/chemokines in CVD although, obviously, the potential contribution of additional cellular sources to the production of plasmatic cytokines/chemokines is not excluded.

In order to evaluate potential anti-inflammatory compounds able to affect the release of this specific set of cytokines/chemokines, we have used $\alpha$-Lipoic acid and Ginkgo biloba derivatives, taking into account previous studies, which have demonstrated that both compounds show antiinflammatory activity on a variety of endothelial cell types [23-35], although they have never been tested before on pathological VEC. The anti-inflammatory activity of both $\alpha$-Lipoic acid and Ginkgo biloba derivatives was confirmed on the basis of their ability to markedly inhibit the surface levels of ICAM-1 and VCAM-1, which are known to promote recruitment of leukocytes, platelets, and erythrocytes to the vein wall $[37,38]$, without inducing toxic effect of VEC cultures. We could demonstrate that, among the cytokines/chemokines found elevated in the plasma of CVD patients and whose in vitro release increased in response to recombinant TNF- $\alpha, \alpha$-Lipoic acid significantly $(P<$ $0.05)$ decreased the basal release of PDGF, RANTES, and CXCL10 and the TNF- $\alpha$-induced release of PDGF, RANTES,
CXCL10, G-CSF, and GM-CSF. On the other hand, Ginkgoselect phytosome decreased the release only of basal PDGF and the TNF- $\alpha$-induced PDGF, RANTES, and CXCL10. Thus, $\alpha$-Lipoic acid showed a broader and more potent inhibitory activity on the release of cytokines/chemokines with respect to Ginkgoselect phytosome. Since recombinant TNF- $\alpha$ induced several intracellular pathways in VEC, such as the JNK, p38/MAPK, ERK1/2, NF- $\kappa$ B, Akt, STAT3, and CREB pathways, it is noteworthy that $\alpha$-Lipoic acid significantly inhibited TNF- $\alpha$-induced NF- $\kappa$ B and dampened p38/MAPK activation, while Ginkgoselect phytosome downmodulated TNF- $\alpha$-induced p38/MAPK and Akt activation. Consistently with a prominent role of NF- $\kappa \mathrm{B}$ and to a lesser extent p38/MAPK pathway in promoting the release of a specific set of cytokines/chemokines in response to TNF$\alpha$, Parthenolide, a pharmacological inhibitor of the NF- $\kappa \mathrm{B}$ pathway, inhibited the release of PDGF, RANTES, CXCL10, G-CSF, and GM-CSF, recapitulating the effect of $\alpha$-Lipoic acid, while MK-2206, a pharmacological inhibitor of the Akt pathway, inhibited the release of PDGF, RANTES, and CXCL10, recapitulating the effect of Ginkgoselect phytosome.

In conclusion, we have demonstrated that pathological VEC significantly contribute to the circulating cytokines/ chemokines found elevated in plasma of CVD patients and by using both $\alpha$-Lipoic acid and Ginkgoselect phytosome, we have characterized and targeted the prominent pathways promoting the release of these cytokines and chemokines.

\section{Conflict of Interests}

The authors declare that there is no conflict of interests regarding the publication of this paper.

\section{Authors' Contribution}

Veronica Tisato and Giorgio Zauli equally contributed to this paper.

\section{Acknowledgments}

The authors thank LABOREST S.p.A. for kindly providing $\alpha$-Lipoic acid and Ginkgoselect phytosome. This work was supported by the Italian Ministry of Health (Project GR-20102310832) and by Regione Emilia Romagna-Programma di ricerca Regione-Università 2010-2012 Strategic Programme (Project PRUA1RI-2012-005).

\section{References}

[1] L. Rochette, S. Ghibu, C. Richard, M. Zeller, Y. Cottin, and C. Vergely, "Direct and indirect antioxidant properties of $\alpha$-lipoic acid and therapeutic potential," Molecular Nutrition \& Food Research, vol. 57, no. 1, pp. 114-125, 2013.

[2] A. Goraca, H. Huk-Kolega, A. Piechota, P. Kleniewska, E. Ciejka, and B. Skibska, "Lipoic acid-biological activity and therapeutic potential," Pharmacological Reports, vol. 63, no. 4, pp. 849-858, 2011.

[3] S. V. Harding, T. C. Rideout, and P. J. Jones, "Evidence for using alpha-lipoic acid in reducing lipoprotein and inflammatory 
related atherosclerotic risk," Journal of Dietary Supplements, vol. 9, no. 2, pp. 116-127, 2012.

[4] S. Golbidi, S. A. Ebadi, and I. Laher, "Antioxidants in the treatment of diabetes," Current Diabetes Reviews, vol. 7, no. 2, pp. 106-125, 2011.

[5] Y.-X. Liu, G.-Z. Han, T. Wu et al., "Protective effect of $\alpha$ lipoic acid on oxidized low density lipoprotein-induced human umbilical vein endothelial cell injury," Pharmacological Reports, vol. 63, no. 5, pp. 1180-1188, 2011.

[6] S. V. Pierre, P. Lesnik, M. Moreau et al., "The standardized Ginkgo biloba extract Egb-761 protects vascular endothelium exposed to oxidized low density lipoproteins," Cellular \& Molecular Biology, vol. 54, pp. OL1032-OL1042, 2008.

[7] T. W. Wakefield, R. M. Strieter, M. R. Prince, L. J. Downing, and L. J. Greenfield, "Pathogenesis of venous thrombosis: a new insight," Cardiovascular Surgery, vol. 5, no. 1, pp. 6-15, 1997.

[8] R. T. Eberhardt and J. D. Raffetto, "Chronic venous insufficiency," Circulation, vol. 111, no. 18, pp. 2398-2409, 2005.

[9] A. M. Müller, M. I. Hermanns, C. Cronen, and C. J. Kirkpatrick, "Comparative study of adhesion molecule expression in cultured human macro- and microvascular endothelial cells," Experimental and Molecular Pathology, vol. 73, no. 3, pp. 171$180,2002$.

[10] I. Lang, M. A. Pabst, U. Hiden et al., "Heterogeneity of microvascular endothelial cells isolated from human term placenta and macrovascular umbilical vein endothelial cells," European Journal of Cell Biology, vol. 82, no. 4, pp. 163-173, 2003.

[11] E. E. Eriksson, E. Karlof, K. Landmark, P. Rotzius, U. Hedin, and X. Xie, "Powerful inflammatory properties of large vein endothelium in vivo," Arteriosclerosis, Thrombosis, and Vascular Biology, vol. 25, no. 4, pp. 723-728, 2005.

[12] M. A. Gonzalez and A. P. Selwyn, "Endothelial function, inflammation, and prognosis in cardiovascular disease," The American Journal of Medicine, vol. 115, no. 8, supplement 1, pp. 99S-106S, 2003.

[13] M. H. Murad, F. Coto-Yglesias, M. Zumaeta-Garcia et al., "A systematic review and meta-analysis of the treatments of varicose veins," Journal of Vascular Surgery, vol. 53, no. 5, supplement, pp. 49S-65S, 2011.

[14] V. Tisato, G. Zauli, R. Voltan et al., "Endothelial cells obtained from patients affected by chronic venous disease exhibit a proinflammatory phenotype," PLoS One, vol. 7, no. 6, Article ID e39543, 2012.

[15] G. Zauli, F. Corallini, F. Bossi et al., "Osteoprotegerin increases leukocyte adhesion to endothelial cells both in vitro and in vivo," Blood, vol. 110, no. 2, pp. 536-543, 2007.

[16] S. Indraccolo, L. Moserle, V. Tisato et al., "Gene therapy of ovarian cancer with IFN- $\alpha$-producing fibroblasts: comparison of constitutive and inducible vectors," Gene Therapy, vol. 13, no. 12, pp. 953-965, 2006.

[17] M. Vitale, L. Zamai, E. Falcieri, G. Zauli, P. Gobbi, and S. Santi, "IMP dehydrogenase inhibitor, tiazofurin, induces apoptosis in K562 human erythroleukemia cells," Cytometry, vol. 30, no. 1, pp. 61-66, 1997.

[18] G. Zauli, G. Visani, A. Bassini et al., "Nuclear translocation of protein kinase C- $\alpha$ and $-\zeta$ isoforms in HL- 60 cells induced to differentiate along the granulocytic lineage by all-trans retinoic acid," British Journal of Haematology, vol. 93, no. 3, pp. 542-550, 1996.

[19] G. Zauli, B. Toffoli, M. G. Di Iasio, C. Celeghini, B. Fabris, and P. Secchiero, "Treatment with recombinant tumor necrosis factor-related apoptosis-inducing ligand alleviates the severity of streptozotocin-induced diabetes," Diabetes, vol. 59, no. 5, pp. 1261-1265, 2010.

[20] G. Zauli, A. Bassini, M. Vitale et al., “Thrombopoietin enhances the alpha IIb beta 3-dependent adhesion of megakaryocytic cells to fibrinogen or fibronectin through PI 3 kinase," Blood, vol. 89, no. 3, pp. 883-895, 1997.

[21] P. Borgatti, M. Mazzoni, C. Carini et al., "Changes of nuclear protein kinase $\mathrm{C}$ activity and isotype composition in PC12 cell proliferation and differentiation," Experimental Cell Research, vol. 224, no. 1, pp. 72-78, 1996.

[22] D. Milani, M. Mazzoni, P. Borgatti, G. Zauli, L. Cantley, and S. Capitani, "Extracellular human immunodeficiency virus type1 Tat protein activates phosphatidylinositol 3-kinase in PC12 neuronal cells," Journal of Biological Chemistry, vol. 271, no. 38, pp. 22961-22964, 1996.

[23] W.-J. Zhang and B. Frei, " $\alpha$-Lipoic acid inhibits TNF- $\alpha$-induced $\mathrm{NF}-\kappa \mathrm{B}$ activation and adhesion molecule expression in human aortic endothelial cells," The FASEB Journal, vol. 15, no. 13, pp. 2423-2432, 2001.

[24] S. Sola, M. Q. Mir, F. A. Cheema et al., "Irbesartan and lipoic acid improve endothelial function and reduce markers of inflammation in the metabolic syndrome: results of the Irbesartan and Lipoic Acid in Endothelial Dysfunction (ISLAND) study," Circulation, vol. 111, no. 3, pp. 343-348, 2005.

[25] B. B. Heinisch, M. Francesconi, F. Mittermayer et al., "Alphalipoic acid improves vascular endothelial function in patients with type 2 diabetes: a placebo-controlled randomized trial," European Journal of Clinical Investigation, vol. 40, no. 2, pp. 148$154,2010$.

[26] G. Xiang, J. Pu, L. Yue, J. Hou, and H. Sun, “ $\alpha$-lipoic acid can improve endothelial dysfunction in subjects with impaired fasting glucose," Metabolism, vol. 60, no. 4, pp. 480-485, 2011.

[27] D. W. Wray, S. K. Nishiyama, R. A. Harris et al., "Acute reversal of endothelial dysfunction in the elderly after antioxidant consumption," Hypertension, vol. 59, no. 4, pp. 818-824, 2012.

[28] S. T. Rahman, N. Merchant, T. Haque, J. Wahi, S. Bhaheetharan, and K. C. Ferdinand, "The impact of lipoic acid on endothelial function and proteinuria in quinapril-treated diabetic patients with stage I hypertension: results from the QUALITY study," Journal of Cardiovascular Pharmacology and Therapeutics, vol. 17, no. 2, pp. 139-145, 2012.

[29] J.-W. Chen, Y.-H. Chen, F.-Y. Lin, Y.-L. Chen, and S.-J. Lin, "Ginkgo biloba extract inhibits tumor necrosis factor$\alpha$-induced reactive oxygen species generation, transcription factor activation, and cell adhesion molecule expression in human aortic endothelial cells," Arteriosclerosis, Thrombosis, and Vascular Biology, vol. 23, no. 9, pp. 1559-1566, 2003.

[30] S. Zhang, B. Chen, W. Wu, L. Bao, and R. Qi, "Ginkgolide $\mathrm{B}$ reduces inflammatory protein expression in oxidized lowdensity lipoprotein-stimulated human vascular endothelial cells," Journal of Cardiovascular Pharmacology, vol. 57, no. 6, pp. 721-727, 2011.

[31] R. Xie, X. Li, Y. Ling, C. Shen, X. Wu, and W. Xu, "Alphalipoic acid pre- and post-treatments provide protection against in vitro ischemia-reperfusion injury in cerebral endothelial cells via Akt/mTOR signaling," Brain Research, vol. 1482, pp. 81-90, 2012.

[32] P. Larghero, R. Venè, S. Minghelli et al., "Biological assays and genomic analysis reveal lipoic acid modulation of endothelial cell behavior and gene expression," Carcinogenesis, vol. 28, no. 5, pp. 1008-1020, 2007. 
[33] M. Artwohl, K. Muth, K. Kosulin et al., "R-(+)- $\alpha$-lipoic acid inhibits endothelial cell apoptosis and proliferation: involvement of Akt and retinoblastoma protein/E2F-1," American Journal of Physiology-Endocrinology and Metabolism, vol. 293, no. 3, pp. E681-E689, 2007.

[34] H.-C. Ou, W.-J. Lee, I.-T. Lee et al., "Ginkgo biloba extract attenuates oxLDL-induced oxidative functional damages in endothelial cells," Journal of Applied Physiology, vol. 106, no. 5, pp. 1674-1685, 2009.

[35] C.-L. Hsu, Y.-L. Wu, G.-J. Tang, T.-S. Lee, and Y. R. Kou, “Ginkgo biloba extract confers protection from cigarette smoke extractinduced apoptosis in human lung endothelial cells: role of heme oxygenase-1," Pulmonary Pharmacology \& Therapeutics, vol. 22, no. 4, pp. 286-296, 2009.

[36] Z.-G. Liu, "Molecular mechanism of TNF signaling and beyond," Cell Research, vol. 15, no. 1, pp. 24-27, 2005.

[37] C. Lawson and S. Wolf, "ICAM-1 signaling in endothelial cells," Pharmacological Reports, vol. 61, no. 1, pp. 22-32, 2009.

[38] P. Alcaide, E. Maganto-Garcia, G. Newton et al., "Difference in Th1 and Th17 lymphocyte adhesion to endothelium," The Journal of Immunology, vol. 188, no. 3, pp. 1421-1430, 2012. 


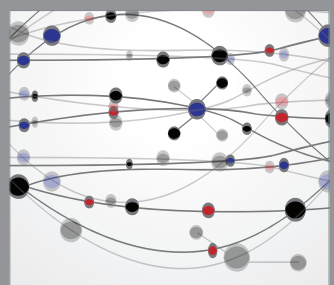

The Scientific World Journal
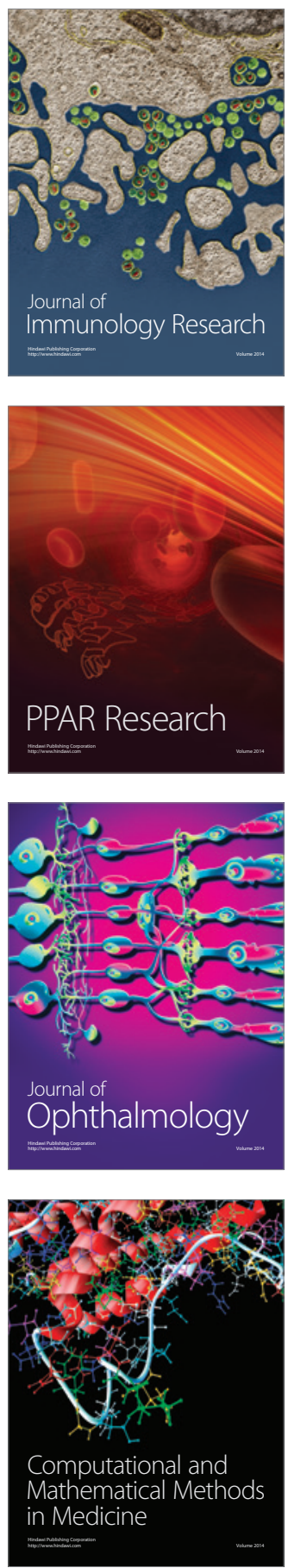

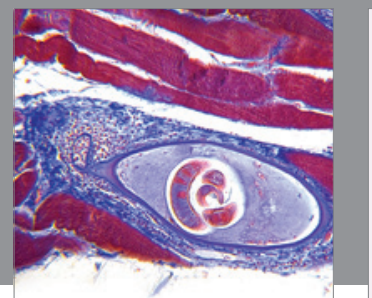

Gastroenterology

Research and Practice
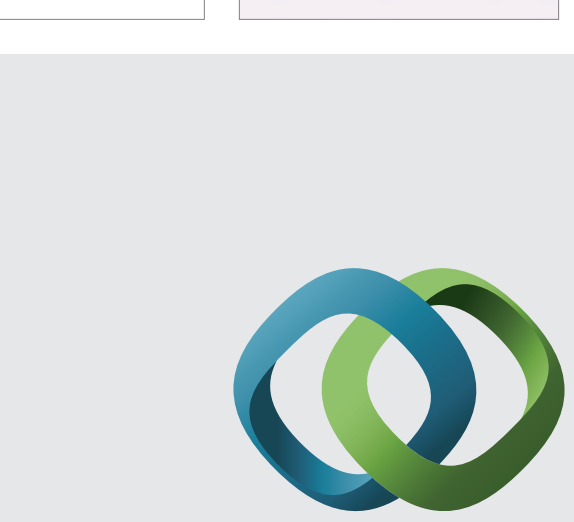

\section{Hindawi}

Submit your manuscripts at

http://www.hindawi.com
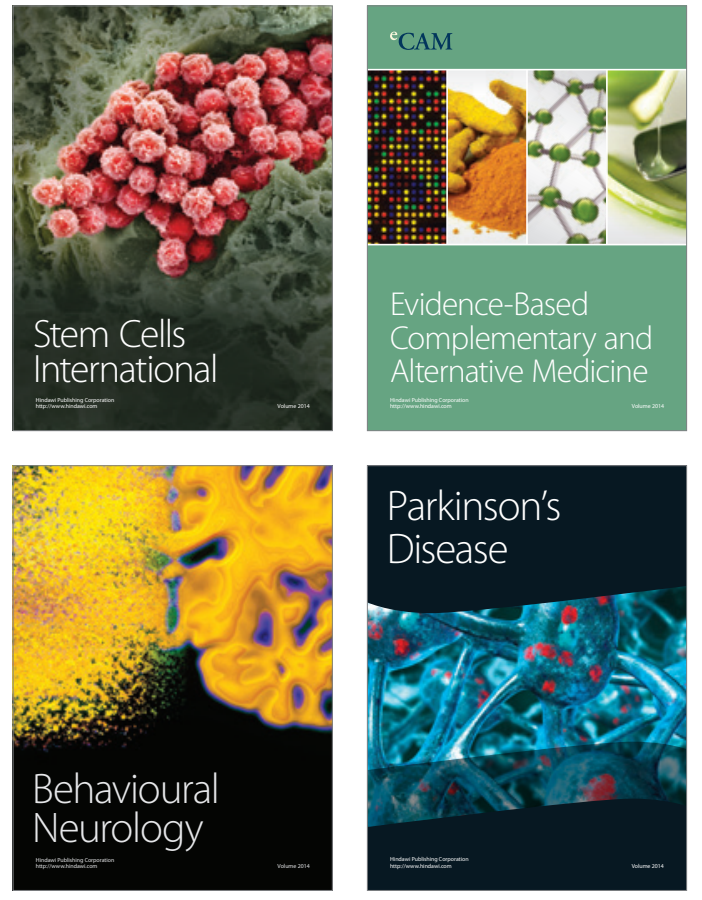
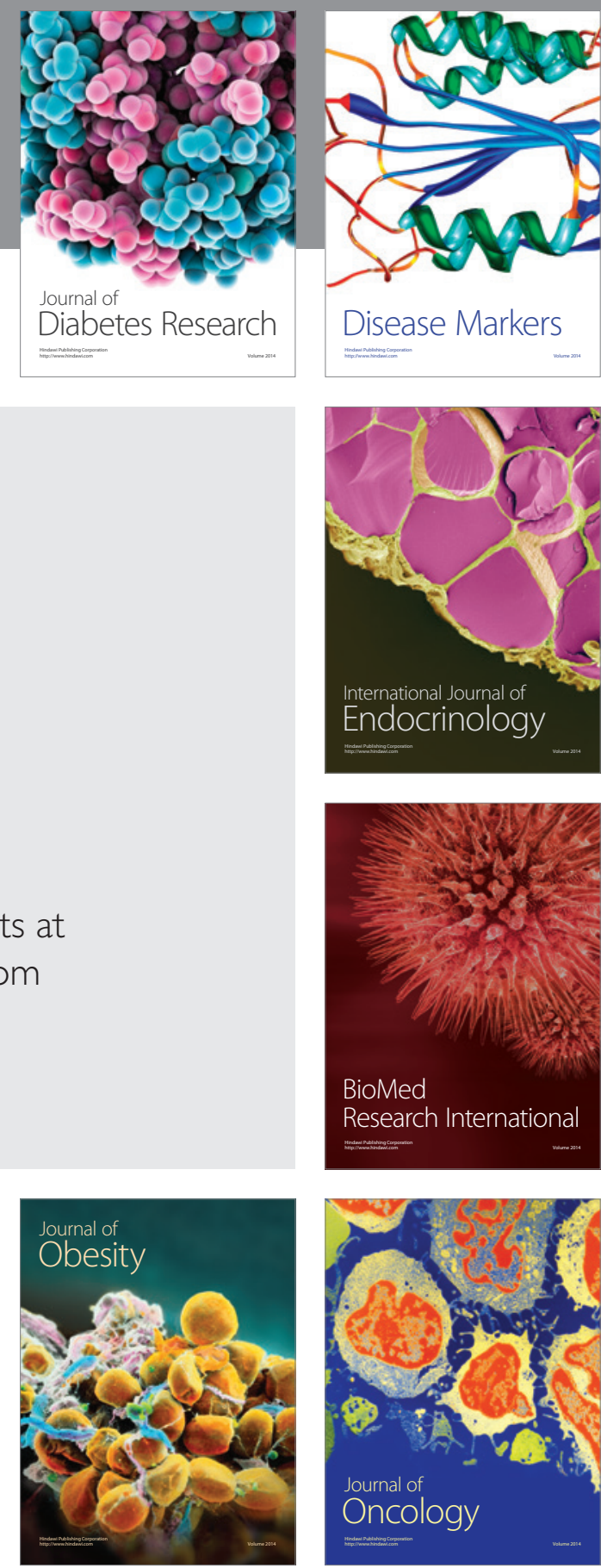

Disease Markers
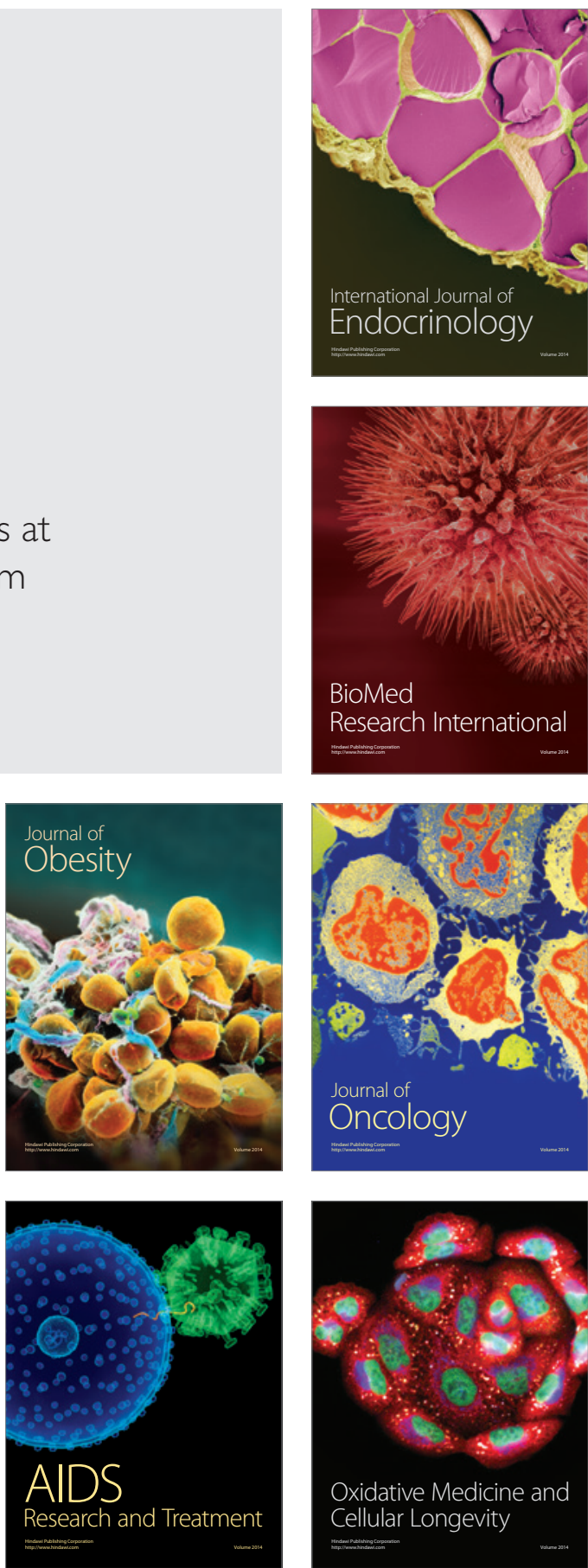